\title{
Comparison of immunochromatographic assays based on fluorescent microsphere and quantum-dot submicrobead for quantitative detection of aflatoxin $M_{1}$ in milk
}

\author{
Chenghui Wu, ${ }^{* 1}$ Liming Hu, ${ }^{* 1}$ Jun Xia,† Guomao Xu,† Kai Luo, ${ }^{*}$ Daofeng Liu, † Hong Duan, ${ }^{*}$ Song Cheng, \\ Yonghua Xiong, ${ }^{*}$ and Weihua Lai ${ }^{* 2}$ \\ *State Key Laboratory of Food Science and Technology, Nanchang University, Nanchang 330047, China \\ †Jiangxi Institute of Veterinary Drug and Feedstuff Control, Nanchang 330047, China \\ łJiangxi Province Center for Disease Control and Prevention, Nanchang 330047, China
}

\begin{abstract}
The performance of fluorescent microsphere immunochromatographic assay (FM-ICA) and quantum-dot submicrobead immunochromatographic assay (QBICA) was systematically and comprehensively compared in quantitative detection of aflatoxin $\mathrm{M}_{1}$ in milk. Under optimum conditions, the advantages of FM-ICA include lower limit of detection of $42.3 \mathrm{pg} / \mathrm{mL}$ with better accuracy, precision, reliability, and practicability. The advantages of QB-ICA include shorter detection time and lower monoclonal antibody consumption. The 2 ICA were consistent with liquid chromatographytandem mass spectrometry. This study serves as a reference for selecting appropriate fluorescent labels for the immunochromatographic assay of aflatoxin $\mathrm{M}_{1}$.

Key words: immunochromatographic assay, fluorescent microsphere, quantum-dot submicrobead, milk
\end{abstract}

\section{INTRODUCTION}

Aflatoxin $\mathrm{M}_{1}$ (AFM1) is a major monohydroxylated derivative of aflatoxin $\mathrm{B}_{1}$ (AFB1; Guo et al., 2014), which is produced by cytochrome $\mathrm{P} 450$-associated enzymes in the liver and also known as "milk toxin" (Taherimaslak et al., 2014). Aflatoxin $\mathrm{M}_{1}$ has been classified as a group I carcinogenic agent by the International Agency for Research on Cancer in 2002 (Du et al., 2016). Various analytical methods have been developed to detect AFM1. These methods include mass spectrometry (Busman et al., 2015), liquid chromatography (Huang et al., 2015), and ultra-high performance

\footnotetext{
Received September 28, 2016.

Accepted December 12, 2016.

${ }^{1}$ These authors contributed equally to this study.

${ }^{2}$ Corresponding author: talktolaiwh@163.com
}

liquid chromatography-tandem mass spectrometry (Ossa et al., 2015). These methods are highly specific and sensitive but require professional operator training and expensive apparatus. Furthermore, the pretreatment of samples is time consuming and unsuitable for on-site detection.

Compared with the above-mentioned methods, immunoassay has attracted attention because of its high sensitivity, high selectivity, simple sample extraction processes, and cost effectiveness (Vdovenko et al., 2014). Immunochromatographic assay (ICA) is a widely used immunoassay. Colloidal gold (CG) is conventionally used as label in ICA because a gold-nanoparticle-based assay is cheap and fast. However, the detection sensitivity of CG-ICA is limited (Shan et al., 2015). In recent years, fluorescent microsphere (FM) and quantum dot (QD) instead of CG have been developed and used in ICA format because of their high sensitivity and accuracy.

Fluorescent microsphere-labeled immunochromatographic assay (FM-ICA) has recently been widely applied in medical diagnosis (Nankoberanyi et al., 2014; Wang et al., 2015; Yang et al., 2015), food safety (Liu et al., 2015), animal health (Huang et al., 2013; Zhang et al., 2016), and agriculture (Chen et al., 2013a; Zhou et al., 2014). Antibodies chemically bind to the surfaces of FM, thus providing a high degree of long-term stability in sample detection. Different from CG-ICA, FM-ICA possesses high sensitivity, good thermal stability, and high FI (Wang et al., 2014).

Quantum dots have attracted great interest as nanoscale fluorescent labels because of their interesting optical and electronic properties, broad UV excitation, narrow fluorescent emission spectra, high quantum yield, large Stoke's shifts, and high photochemical stability (Zhang et al., 2014; Ensafi et al., 2016; Lu et al., 2016). Furthermore, QD submicrobead (QB), in which numerous QD are embedded in a polymer matrix, im- 
prove the sensitivity of immunoassay because of their stronger FI than the corresponding single QD. Many recent studies have used QB-ICA for the ultrasensitive detection of analytes in medical diagnosis ( $\mathrm{Li}$ et al., 2014; Shen et al., 2015; Hu et al., 2016) and food safety (Duan et al., 2015). They found that QB-ICA has significantly higher sensitivity than the corresponding single QD-ICA.

Xie et al. (2014) and Zhang et al. (2015) compared the use of FM with that of CG in label-based ICA. Meanwhile, Ren et al. (2014) compared the performance of QB with that of the corresponding single QD in label-based ICA. However, a comparison of performance between FM- and QB-based ICA has yet to be conducted. The performance of FM-ICA and QB-ICA in quantitative detection of AFM1 was systematically and comprehensively compared for the first time in the present study. The detection principle based on competitive inhibition is shown in Figure 1. This study serves as a reference for selecting appropriate fluorescent labels for ICA in the detection of AFM1 in milk.

\section{MATERIALS AND METHODS}

\section{Materials and Reagents}

The FM was purchased from Merck Company (Darmstadt, Germany). The QB was purchased from Ocean NanoTech (San Diego, CA). The AFM1 standard solutions, $N$-(3-dimethylaminopropyl)- $N$-ethylcarbodiimide hydrochloride (EDC·HCl), and BSA were obtained from Sigma-Aldrich (St. Louis, MO). Donkey anti-mouse antibody, anti-AFM1 monoclonal antibody ( $\mathbf{m A b})$, and AFM1-BSA conjugate with a molar substitution ratio of 40:1 were obtained from Zodolabs Bioengineering Co. Ltd. (Nanchang, Jiangxi, China). Nitrocellulose membrane, absorbent pad, and sample pad were purchased from Millipore (Bedford, MA). Milk samples were purchased from Rainbow Supermarket (Nanchang, Jiangxi, China). All other chemicals and reagents were of analytical grade and purchased from Sinopharm Chemical Reagent Co. Ltd. (Shanghai, China).
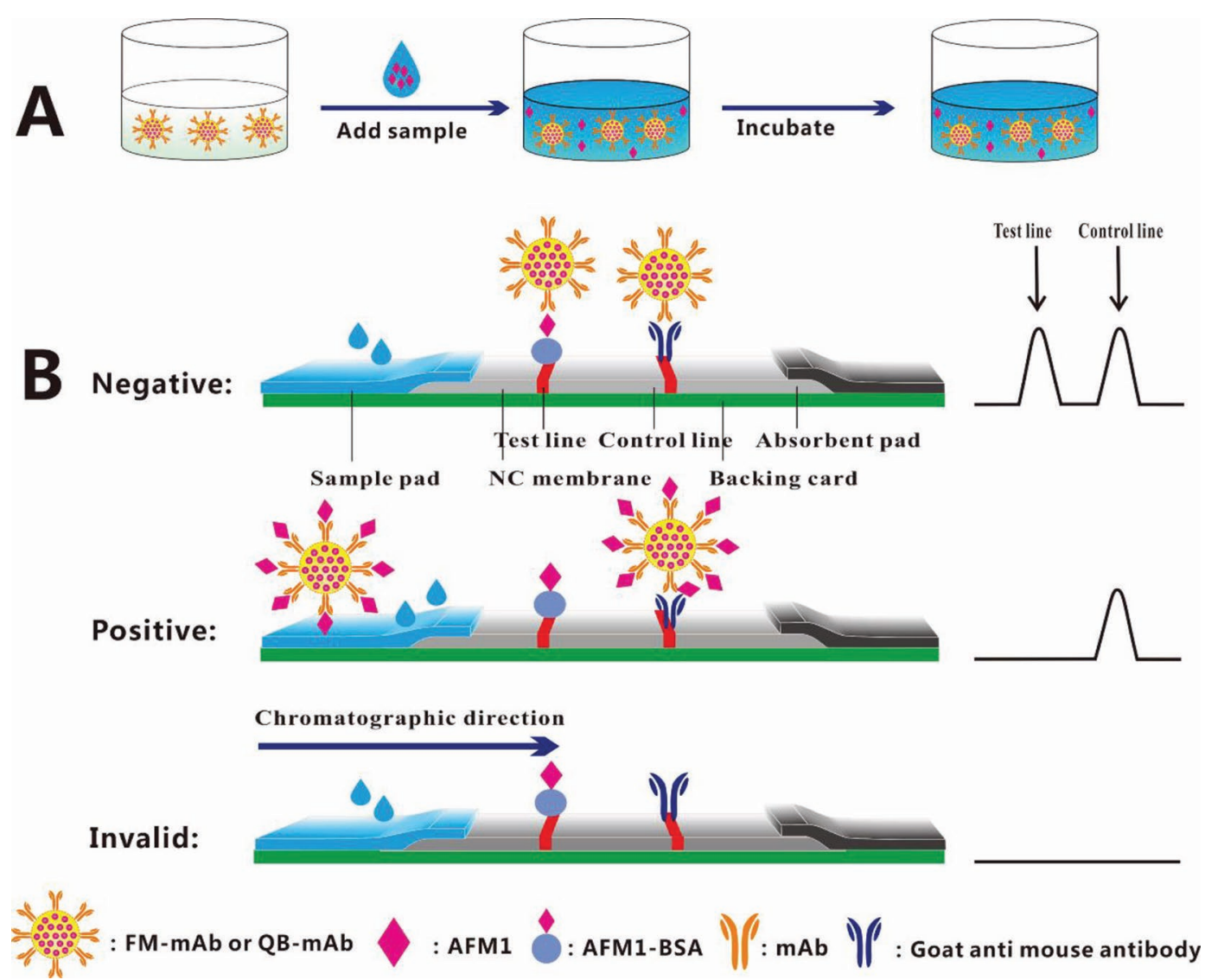

Figure 1. Schematic diagram of fluorescent microsphere immunochromatographic assay (FM-ICA) and quantum-dot submicrobead immunochromatographic assay (QB-ICA) for quantitative detection of aflatoxin $\mathrm{M}_{1}$ (AFM1) in milk. A: Pre-incubation of milk samples and FM/ QB-monoclonal antibody (mAb) probe in ELISA well for 10 min. B: Detection principle of ICA strips. NC = nitrocellulose. 


\section{Apparatus}

The FM-ICA reader (excitation wavelength $=470$ $\mathrm{nm}$; emission wavelength $=520 \mathrm{~nm}$ ) and QB-ICA reader (excitation wavelength $=365 \mathrm{~nm}$; emission wavelength $=620$ ) were from Zhejiang Fenghang Science Instrument Co. Ltd. (Zhejiang, China). The BioDot XYZ platform equipped with a motion controller and Guillotine Cutting Module were supplied by BioDot (Irvine, CA). A vacuum drying oven was purchased from Shanghai Fumma Laboratory Instrument Co. Ltd. (Shanghai, China). A high-speed freezing centrifuge was purchased from Xiang Yi (Hunan, China).

\section{Key Parameters in Optimization of FM-ICA and Preparation of the Test Strip}

Preparation of FM-mAb Probe. An FM-mAb probe was prepared as follows. In brief, $6 \mu \mathrm{g}$ of antiAFM1 antibody was added to $2.6 \mathrm{~mL}$ of $0.01 \mathrm{M}$ phosphate buffer with different $\mathrm{pH}$ values $(5.0,6.0,7.0,8.0$, 9.0, and 10.0) and containing $0.15 \mathrm{mg}$ of FM and 2.7 $\mu \mathrm{L}$ of $\mathrm{EDC} \cdot \mathrm{HCl}(5 \mathrm{mg} / \mathrm{mL})$. The solution was agitated for $2 \mathrm{~h}$. Subsequently, $300 \mu \mathrm{L}$ of BSA (10\%, wt/vol) was added as a blocking buffer, and the solution was agitated for $30 \mathrm{~min}$. After centrifugation $(19,460 \times$ $g$ at $4^{\circ} \mathrm{C}$ for $10 \mathrm{~min}$ ), the supernatant was discarded, and the precipitate was resuspended in a $300-\mu \mathrm{L}$ solution containing $0.02 \mathrm{~mol} / \mathrm{L}$ of $\mathrm{Na}_{2} \mathrm{HPO}_{4}$ at $\mathrm{pH} 5.5,5 \%$ sucrose, $3 \%$ trehalose, $0.1 \% \mathrm{NaN}_{3}, 1 \% \mathrm{BSA}$, and $1 \%$ Tween-20. Dynamic light scattering (DLS) analysis of the free FM and FM-mAb probe was performed using the particle size analyzer to confirm the immobilization of anti-AFM1 mAb on the FM surface.

Optimizing the Volume of the FM-mAb Probe. Standard AFM1 milk solutions (98.2, 97.8, and 97.4 $\mu \mathrm{L})$ were mixed with the FM-mAb probe $(1.8,2.2$, and $2.6 \mu \mathrm{L}$ ) in the ELISA well to determine the influence of $\mathrm{FM}-\mathrm{mAb}$ probe volume on the fluorescent intensity of the $\mathrm{T}$ line $\left(\mathbf{F I}_{\mathbf{T}}\right)$, the fluorescent intensity of the $\mathrm{C}$ line $\left(\mathbf{F I}_{\mathrm{C}}\right), \mathrm{FI}_{\mathrm{T}} / \mathrm{FI}_{\mathrm{C}}$, and positive inhibition rate (500 $\mathrm{pg} / \mathrm{mL}$ ). The mixture was then transferred to the FMICA strips. After $35 \mathrm{~min}$ of immune reaction, the $\mathrm{FI}_{\mathrm{T}}$, $\mathrm{FI}_{\mathrm{C}}$, and $\mathrm{FI}_{\mathrm{T}} / \mathrm{FI}_{\mathrm{C}}$ were recorded by the fluorescent strip reader.

Assembling the FM-ICA Test Strip. The AFM1-BSA $(0.15,0.25$, and $0.3 \mathrm{mg} / \mathrm{mL})$ and donkey anti-mouse antibody $(0.55 \mathrm{mg} / \mathrm{mL})$ were sprayed onto the nitrocellulose membrane to form the $\mathrm{T}$ and $\mathrm{C}$ lines, respectively. The prepared nitrocellulose membrane and the absorption, conjugate, and sample pads were assembled to prepare the FM-ICA test strip.

\section{Optimization of Key Parameters for QB-ICA and Preparation of Test Strip}

Preparation of $Q B-m A b$ Probe. A QB-mAb probe was prepared as follows. In brief, $0.5 \mu \mathrm{g}$ of $\mathrm{EDC} \cdot \mathrm{HCl}, 0.06 \mathrm{mg}$ of QB, and $6 \mu \mathrm{g}$ of anti-AFM1 $\mathrm{mAb}$ were added to $180 \mu \mathrm{L}$ of $0.01 M$ phosphate buffer with different $\mathrm{pH}$ values $(5.0,6.0,7.0,8.0,9.0$, and 10.0). After reacting at room temperature for $1 \mathrm{~h}$ under magnetic stirring, the mixtures were centrifuged at 18,070 $\times g$ at $4^{\circ} \mathrm{C}$ for $10 \mathrm{~min}$. The $\mathrm{QB}$ and anti-AFM $1 \mathrm{mAb}$ conjugates were resuspended with $180 \mu \mathrm{L}$ of PBS $(0.01$ $M, \mathrm{pH} 7.4$ ) containing $2 \%$ fructose, $1 \%$ polyethylene glycol (PEG 20000, Merck Company), $5 \%$ sucrose, $1 \%$ BSA, and $0.4 \%$ Tween-20. The DLS analysis of the free $\mathrm{QB}$ and $\mathrm{QB}-\mathrm{mAb}$ probe was performed using a particle size analyzer to confirm the immobilization of anti-AFM1 mAb on the QB surface.

Optimizing the Volume of the QB-mAb Probe. Milk samples $(79,78$, and $77 \mu \mathrm{L})$ were mixed with the QB-mAb probe $(1.0,2.0$, and $3.0 \mu \mathrm{L})$ in the ELISA well to determine the $\mathrm{FI}_{\mathrm{T}}, \mathrm{FI}_{\mathrm{C}}, \mathrm{FI}_{\mathrm{T}} / \mathrm{FI}_{\mathrm{C}}$, and positive inhibition rate $(1,000 \mathrm{pg} / \mathrm{mL})$. The mixture was then transferred to the QB-ICA strips. After $15 \mathrm{~min}$ of immune reaction, the $\mathrm{FI}_{\mathrm{T}}, \mathrm{FI}_{\mathrm{C}}$, and $\mathrm{FI}_{\mathrm{T}} / \mathrm{FI}_{\mathrm{C}}$ were recorded by the fluorescent strip reader.

Assembling the QB-ICA Test Strip. The AFM1BSA $(0.05,0.1$, and $0.15 \mathrm{mg} / \mathrm{mL})$ and donkey antimouse antibody $(1.0 \mathrm{mg} / \mathrm{mL})$ were sprayed onto the nitrocellulose membrane to form the $\mathrm{T}$ and $\mathrm{C}$ lines, respectively. The prepared nitrocellulose membrane and the absorption, conjugate, and sample pads were assembled to prepare the QB-ICA strip.

\section{Quantitative Procedure of QB-ICA and FM-ICA}

Standard AFM1 milk solutions were prepared using spiked stock AFM1 solution $(1.0 \mu \mathrm{g} / \mathrm{mL})$ in PBS containing $3.0 \%$ methanol (vol/vol) at $\mathrm{pH} 7.4$ to final concentrations of $0,50,100,200,300,400,500,600$, $700,800,900,1,000,1,100,1,200$, and $1,250 \mathrm{pg} / \mathrm{mL}$. The FM-mAb probe $(2.2 \mu \mathrm{L})$ and the $\mathrm{QB}-\mathrm{mAb}$ probe $(1.0 \mu \mathrm{L})$ were pre-incubated with 97.8 and $79 \mu \mathrm{L}$ of standard AFM1 milk solutions in an ELISA well at room temperature to form the FM-mAb probe mixture and QB-ICA mixture, respectively. After $10 \mathrm{~min}$ of incubation, the FM-mAb probe mixture and QB-ICA mixture were transferred to FM-ICA and QB-ICA, respectively. The strips were scanned with the fluorescent strip reader. The $\mathrm{FI}_{\mathrm{T}}, \mathrm{FI}_{\mathrm{C}}$, and $\mathrm{FI}_{\mathrm{T}} / \mathrm{FI}_{\mathrm{C}}$ were recorded. An AFM1 quantitative analysis was calculated according to the linear regression equation of the AFM1 cali- 
bration curve. The standard curve was established by plotting $\mathrm{B} / \mathrm{B}_{0}$ against the logarithm of the AFM1 concentration, where $\mathrm{B}$ and $\mathrm{B}_{0}$ represent the $\mathrm{FI}_{\mathrm{T}} / \mathrm{FI}_{\mathrm{C}}$ with and without AFM1 in the milk solutions, respectively.

\section{Comparison of FM-ICA and QB-ICA}

Limit of Detection of FM-ICA and QB-ICA. The limit of detection (LOD) was calculated as the average $\mathrm{FI}_{\mathrm{T}} / \mathrm{FI}_{\mathrm{C}}$ of 20 blank milk samples minus 3 standard deviations from the average.

Accuracy and Precision of 2 ICA Strips. The accuracy and precision of FM-ICA were evaluated by analyzing the recovery and coefficients of variation of the intraassays and interassays in 3 spiked milk samples with AFM1 concentrations of 100,300 , and $500 \mathrm{pg} / \mathrm{mL}$. Similarly, the accuracy and precision of QB-ICA were evaluated by analyzing the recovery and coefficients of variation of the intraassays and interassays in 3 spiked milk samples with AFM1 concentrations of 300, 600, and $900 \mathrm{pg} / \mathrm{mL}$. The intraassay was completed within $1 \mathrm{~d}$ with 3 replicates for each spiked concentration, and the interassays were continuously conducted every $3 \mathrm{~d}$ with 3 replicates for each spiked concentration.

Specificity of FM-ICA and QB-ICA. The specificity of FM-ICA and QB-ICA was evaluated by analyzing AFM1 and 4 other mycotoxins, namely, deoxynivalenol (DON), AFB1, ochratoxin A (OTA), and zearalenone (ZEN) at concentration $10 \mathrm{ng} / \mathrm{mL}$.

Liquid Chromatography-Tandem Mass Spectrometry Analysis. The reliability and practicability of FM-ICA and QB-ICA were further confirmed by a triple quadrupole liquid chromatography-tandem mass spectrometry (LC-MS/MS) system (Agilent Technologies, Lexington, MA) composed of triple quad instruments (Agilent 6410B) and an LC system (Agilent 1200 series). The LC-MS/MS operation was performed in accordance with the national food safety standards of China (GB 5413.37, 2010) with some modifications. A $50-\mathrm{mL}$ milk sample was heated to 35 to $37^{\circ} \mathrm{C}$ in a water bath and then centrifuged at $8,340 \times g$, at $25^{\circ} \mathrm{C}$ for 15 min. After centrifugation, all of the supernatant was collected. The treated supernatant was cleaned using an immune affinity column provided by Beijing Rapid Bioscience Co. Ltd. (Beijing, China). The purified AFM1 solution was filtered with a $0.22-\mu \mathrm{m}$ cellulose membrane and further used for LC-MS/MS analysis. The LC-MS/MS system was controlled using MassHunter (Agilent Technologies, Lexington, MA). The chromatographic separation was performed with an Agilent Zorbax Eclipse SB-C18 column $(2.1 \mathrm{~mm} \times 100$ $\mathrm{mm}, 1.8 \mu \mathrm{m})$ that was maintained at $35^{\circ} \mathrm{C}$. The mobile phase consisted of solvent A $(0.1 \%$ formic acid solu- tion) and solvent B (acetonitrile-methanol solution). The gradient elution conditions are as follows: $0 \mathrm{~min}$, $2 \%$ solvent B; 0.5 min, $2 \%$ solvent B; $3.1 \mathrm{~min}, 100 \%$ solvent B; $6.5 \mathrm{~min}, 100 \%$ solvent B; and $6.2 \mathrm{~min}, 100 \%$ solvent $\mathrm{B}$. The flow rate was $0.30 \mathrm{~mL} / \mathrm{min}$, and the injection volume was $10 \mu \mathrm{L}$ in full loop injection mode. Electrospray ionization was performed in positive ion mode. Detection was conducted in multi-ion reaction monitoring mode. High-purity nitrogen gas was used for desolvation, as cone, and as collision gas. The gas temperature was $325^{\circ} \mathrm{C}$, the gas flow rate was $6 \mathrm{~L} / \mathrm{min}$, and the gas pressure was $310,275 \mathrm{~Pa}$. The monitoring ion pairs were chosen as AFM1 m/z 329.1/273.5 (quantitation ions) and 329.1/259.5 (qualitative ions), respectively.

\section{Comparative Evaluation of FM-ICA and QB-ICA with LC-MS/MS}

Comparative Evaluation of FM-ICA with LC$\boldsymbol{M S} \boldsymbol{M S}$. The milk sample confirmed as a negative sample was spiked with AFM1 at 3 concentrations $(50,250$, and $500 \mathrm{pg} / \mathrm{mL})$ and then analyzed using the developed FM-ICA and LC-MS/MS to study the reliability and practicability of FM-ICA with 3 replicates at each spiked concentration.

Comparative Evaluation of QB-ICA with LC$M S / M S$. The milk sample confirmed as a negative sample was spiked with AFM1 at 3 concentrations $(200,500$, and $800 \mathrm{pg} / \mathrm{mL})$ and then analyzed using the developed QB-ICA and LC-MS/MS to study the reliability and practicability of QB-ICA with 3 replicates at each spiked concentration.

\section{RESULTS AND DISCUSSION}

\section{Detection Principle of ICA}

The detection principle of FM/QB-ICA is based on a competitive ICA as shown in the Figure 1. The mixture including milk and probe-antibody complex is transferred to the sample pad of an ICA test strip from the ELISA well. The AFM1 in milk competes with the AFM1-BSA coated on the nitrocellulose membrane for the limited binding sites of the antibody. Then the ICA test strip was detected by ICA reader. The $\mathrm{FI}_{\mathrm{T}}$ is inversely proportional to AFM1 concentration in milk.

\section{Transmission Electron Microscopy Images of $F M$ and $Q B$}

The size and morphology of FM and QB were studied under a high-resolution transmission electron microscope. Figures 2A, 2B, 2C, and 2D illustrated that the 

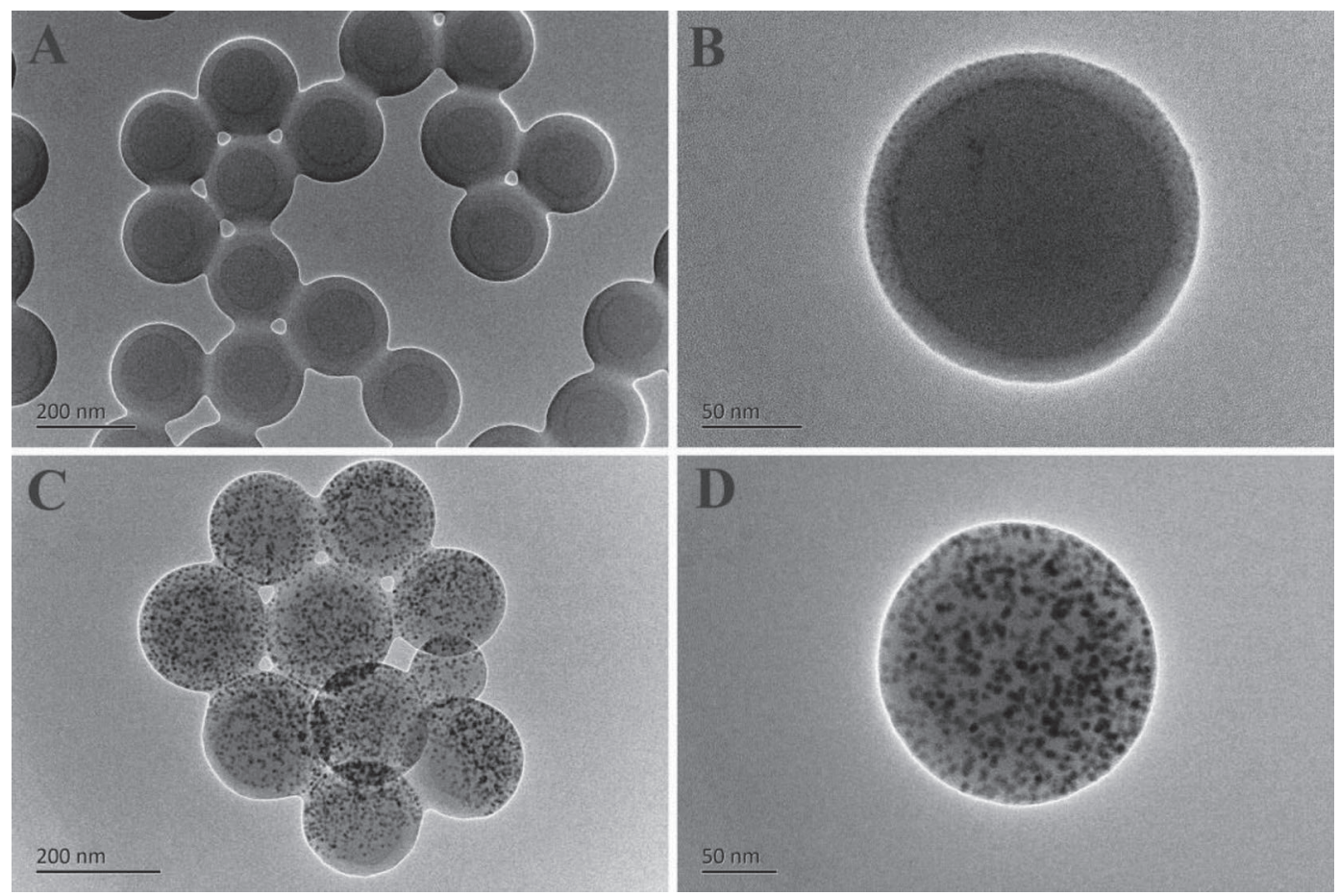

Figure 2. Transmission electron microscopy (TEM) images of fluorescent microsphere (FM) and quantum-dot submicrobead (QB). A and B: TEM images of FM at different magnifications. C and D: TEM images of QB at different magnifications.

FM and QB presented a narrow size distribution and high monodispersity, with respective average sizes of approximately 175 and $250 \mathrm{~nm}$. Furthermore, Figures $2 \mathrm{C}$ and $2 \mathrm{D}$ showed a compact QD-polymer structure, indicating that the numerous individual dark dots were tightly encapsulated in the polymer matrix. The dark dots were the QD, which were visibly identified from the polymer matrix because of the different electron penetrabilities between the QD and the polymer matrix.

\section{Fluorescence Spectra of FM and QB}

The fluorescence spectra of FM and QB were characterized. Figures $3 \mathrm{~A}$ and $3 \mathrm{~B}$ showed that the maximum excitations of FM and QB were at 468 and $395 \mathrm{~nm}$, respectively, whereas their maximized emissions were at 526 and $627 \mathrm{~nm}$, respectively.

\section{$D L S$ of $F M, F M-m A b, Q B$, and $Q B-m A b$}

The DLS analysis results (Figures 3C and 3D) showed that the hydrodynamic diameters of the free FM and QB were approximately $206.1 \pm 8.2$ and $487.3 \pm 15$ $\mathrm{nm}$, respectively, whereas those of the FM-mAb and QB-mAb probes increased to approximately $242.9 \pm$ 5.2 and $513.6 \pm 9.8 \mathrm{~nm}\left(\Delta \mathrm{d}_{\mathrm{FM}}=36.8 \pm 3.0 \mathrm{~nm}, \Delta \mathrm{d}_{\mathrm{QB}}\right.$
$=26.3 \pm 5.2 \mathrm{~nm}$ ), respectively, where $\mathrm{d}=$ the hydrodynamic diameter, $\Delta \mathrm{d}_{\mathrm{FM}}=\mathrm{d}(\mathrm{FM}-\mathrm{mAb})-\mathrm{d}(\mathrm{FM})$, and $\Delta \mathrm{d}_{\mathrm{QB}}=\mathrm{d}(\mathrm{QB}-\mathrm{mAb})-\mathrm{d}(\mathrm{QB})$. Results confirmed that the anti-AFM1 mAb was successfully labeled onto the surfaces of the FM and QB.

\section{Effects of Label pH on ICA Sensitivity}

Optimizing label $\mathrm{pH}$ to develop an ICA is necessary because this parameter can affect antibody activity (Chen et al., 2013b). However, extreme pH exerts adverse effects on antibody activity. The competitive inhibition rate for the $100 \mathrm{pg} / \mathrm{mL}$ positive AFM1-spiked milk samples reached a maximum of $48.70 \%$ in the $\mathrm{pH}$ 7.0 phosphate buffer solution. The results (Figure 4A) indicated that neutral conditions ( $\mathrm{pH}$ 7.0) improved the sensitivity of FM-ICA by promoting anti-AFM1 mAb and AFM1-BSA recognition. Meanwhile, Figure 4B showed that weak acidic conditions ( $\mathrm{pH}$ 6.0) improved the sensitivity of QB-ICA by promoting anti-AFM1 $\mathrm{mAb}$ and AFM1-BSA recognition.

\section{Kinetic Curves of FM-ICA and QB-ICA}

After the FM-ICA and QB-ICA strips were inserted into the FM-ICA and QB-ICA fluorescence readers, respectively, the $\mathrm{FI}_{\mathrm{T}}, \mathrm{FI}_{\mathrm{C}}$, and $\mathrm{FI}_{\mathrm{T}} / \mathrm{FI}_{\mathrm{C}}$ were recorded 

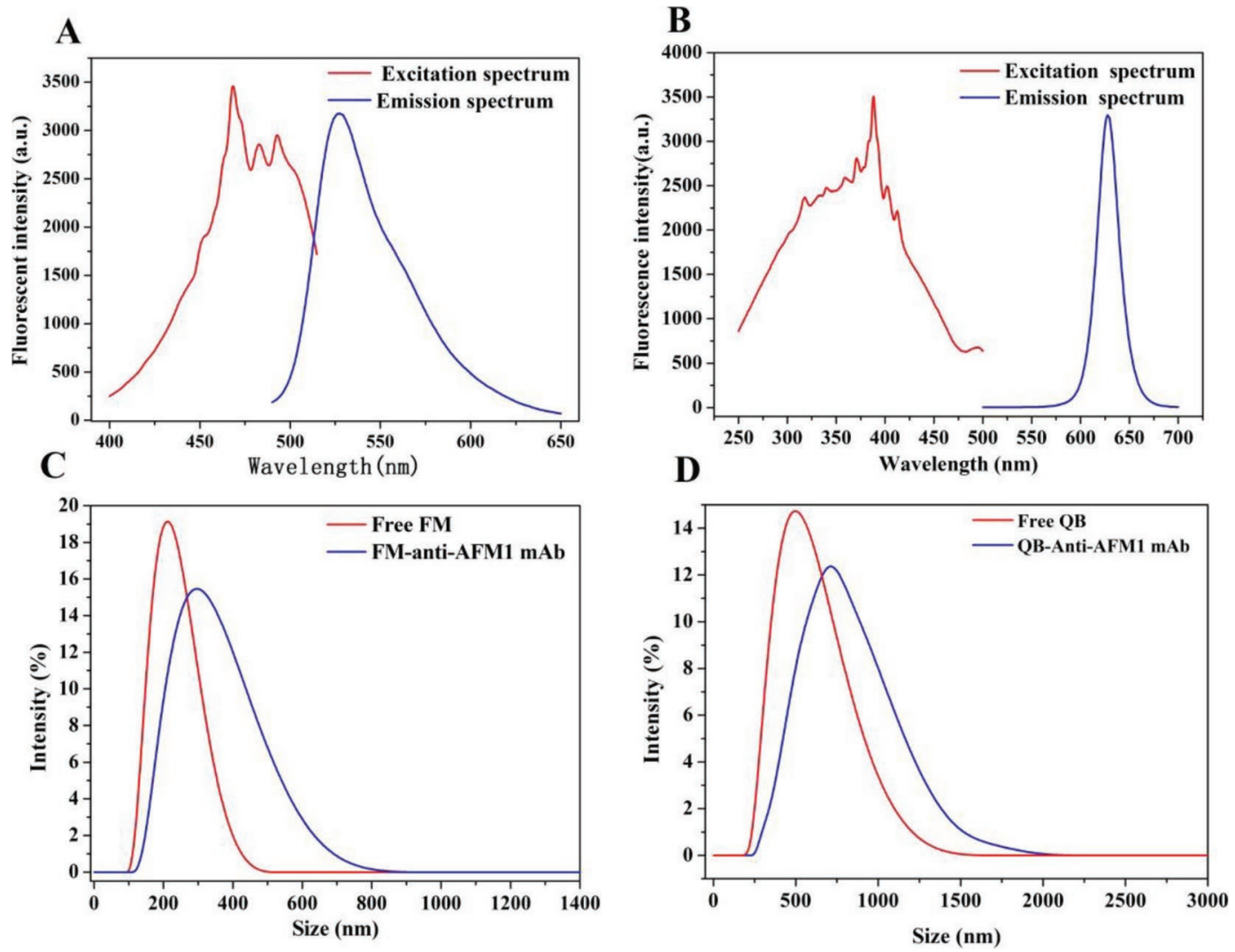

Figure 3. Results of fluorescence spectroscopy and dynamic light scattering. (A) Fluorescence spectra of fluorescent microsphere (FM) excitation and emission. (B) Fluorescence spectra of quantum-dot submicrobead (QB) excitation and emission. (C) Dynamic light scattering analysis of the free FM and FM-monoclonal antibody $(\mathrm{mAb})$ probe. (D) Dynamic light scattering analysis of the free QB and QB-mAb probe. AFM1 = aflatoxin $\mathrm{M}_{1}$. Color version available online.

every 1 min total for $40 \mathrm{~min}$. The kinetic curves were constructed by plotting the values of $\mathrm{FI}_{\mathrm{T}}, \mathrm{FI}_{\mathrm{C}}$, and $\mathrm{FI}_{\mathrm{T}} /$ $\mathrm{FI}_{\mathrm{C}}$ against the immune response time. As shown in Figures $4 \mathrm{C}$ and $4 \mathrm{D}, \mathrm{FI}_{\mathrm{T}}$ and $\mathrm{FI}_{\mathrm{C}}$ continued to increase. However, the $\mathrm{FI}_{\mathrm{T}} / \mathrm{FI}_{\mathrm{C}}$ stabilized at $35 \mathrm{~min}$ (FM-ICA) and 15 min (QB-ICA).

\section{Optimization of Probe Volume and Concentration of AFM1-BSA Antigen}

A similar checkerboard titration was performed with a series of AFM1-BSA concentration on the $\mathrm{T}$ line for different volumes of the FM-mAb and QB-mAb probes to achieve the best sensitivity and appropriate FI on both lines. The FM-ICA and QB-ICA results $\left(\mathrm{FI}_{\mathrm{T}}, \mathrm{FI}_{\mathrm{C}}\right.$, and $\mathrm{FI}_{\mathrm{T}} / \mathrm{FI}_{\mathrm{C}}$ ) were recorded at 35 and 15 min, respectively. The competitive inhibition rates were calculated as follows: $\left(1-\mathrm{B} / \mathrm{B}_{0}\right) \times 100$, where $\mathrm{B}_{0}$ and $\mathrm{B}$ represent the $\mathrm{FI}_{\mathrm{T}} / \mathrm{FI}_{\mathrm{C}}$ of the negative sample and AFM1-spiked milk sample $(500 \mathrm{pg} / \mathrm{mL}$ for FM-ICA and 1,000 pg/
$\mathrm{mL}$ for QB-ICA), respectively. As shown in Table 1, the optimal FM-ICA combinations were as follows: 0.25 $\mathrm{mg} / \mathrm{mL}$ of AFM1-BSA on the T line and $2.2 \mu \mathrm{L}$ of FM$\mathrm{mAb}$ pre-incubated with $77.8-\mu \mathrm{L}$ milk samples. Under the optimal conditions, the means of the $\mathrm{FI}_{\mathrm{T}}$ and $\mathrm{FI}_{\mathrm{C}}$ signals on both lines were $2,984 \pm 33$ and $1,748 \pm 34$, respectively. The competitive inhibition rate for the 500 $\mathrm{pg} / \mathrm{mL}$ spiked milk sample was $89.70 \pm 0.9 \%$. The optimal QB-ICA conditions were as follows: $0.10 \mathrm{mg} / \mathrm{mL}$ AFM1-BSA on the $\mathrm{T}$ line and $1.0 \mu \mathrm{L}$ of $\mathrm{QB}-\mathrm{mAb}$ preincubated with $99-\mu \mathrm{L}$ milk samples. Under the optimal conditions, the means of the $\mathrm{FI}_{\mathrm{T}}$ and $\mathrm{FI}_{\mathrm{C}}$ signal on both lines were $1,625 \pm 190$ and $446 \pm 38$, respectively, and the competitive inhibition rate for the $1,000 \mathrm{pg} / \mathrm{mL}$ spiked milk sample was $82.10 \pm 3.4 \%$.

The following results were obtained under the optimized conditions of FM-ICA and QB-ICA. Up to 0.05 and $0.01 \mu \mathrm{g}$ of $\mathrm{mAb}$ were consumed in FM-ICA and QB-ICA, respectively; the concentration of AFM1-BSA in FM-ICA was 2.5 times that in QB-ICA. 
A
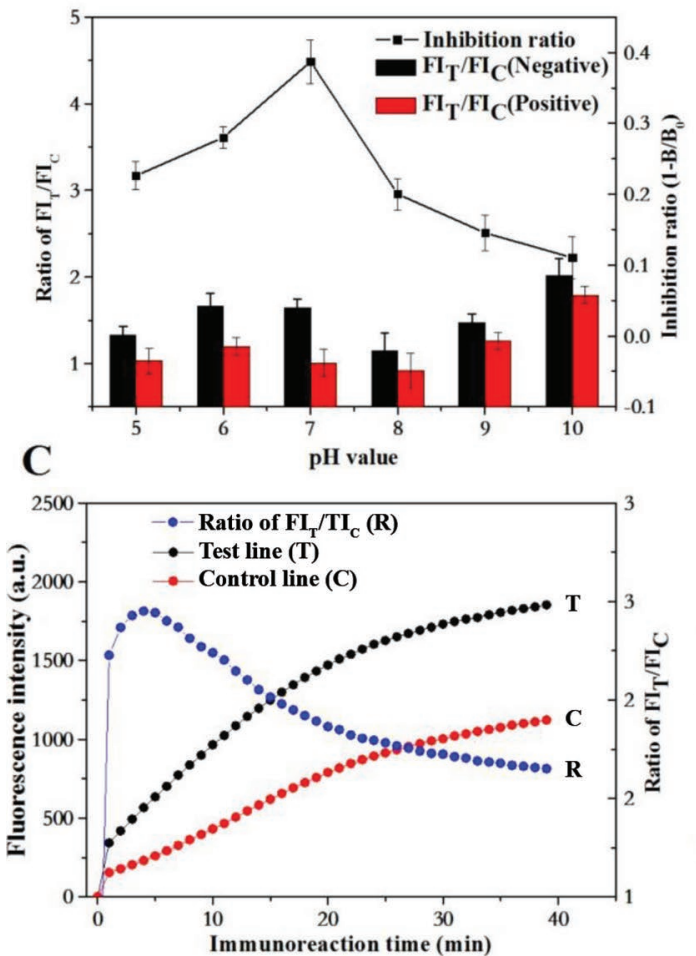

B
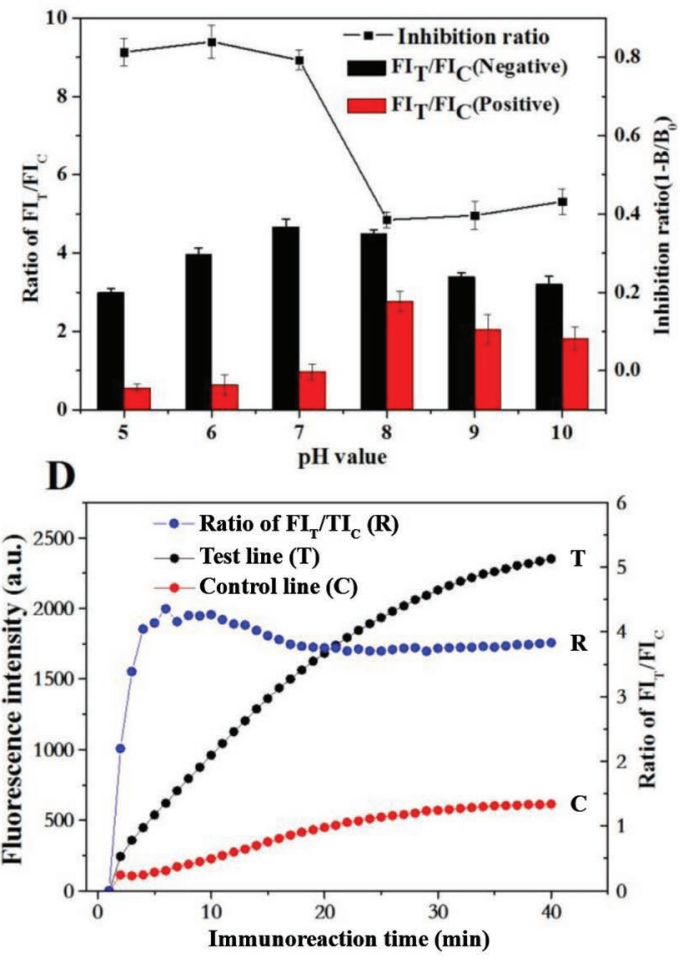

Figure 4. Optimization of labeling $\mathrm{pH}$ and immunoreaction time. A: The effects of labeling $\mathrm{pH}$ on the fluorescent intensity of the $\mathrm{T}$ line $\left(\mathrm{FI}_{\mathrm{T}}\right)$ /fluorescent intensity of the $\mathrm{C}$ line $\left(\mathrm{FI}_{\mathrm{C}}\right)$ and positive $(100 \mathrm{pg} / \mathrm{mL})$ inhibition ratio of fluorescent microsphere immunochromatographic assay (FM-ICA). B and $\mathrm{B}_{0}$ represent the fluorescent intensity of the $\mathrm{T}$ line/fluorescent intensity of the $\mathrm{C}$ line with and without aflatoxin $\mathrm{M}_{1}$ in the milk solutions, respectively. B: The effects of labeling $\mathrm{pH}$ on the $\mathrm{FI}_{\mathrm{T}} / \mathrm{FI}_{\mathrm{C}}$ and positive $(1,000 \mathrm{pg} / \mathrm{mL})$ inhibition ratio of quantum-dot submicrobead immunochromatographic assay (QB-ICA). Error bars indicate SD. C: Immunoreaction dynamics of $\mathrm{FI}_{\mathrm{T}}, \mathrm{FI}_{\mathrm{C}}$, and $\mathrm{FI}_{\mathrm{T}} / \mathrm{FI}_{\mathrm{C}}$ for $\mathrm{FM}_{\mathrm{ICA}}$. a.u. = arbitrary units. D: Immunoreaction dynamics of $\mathrm{FI}_{\mathrm{T}}, \mathrm{FI}_{\mathrm{C}}$, and $\mathrm{FI}_{\mathrm{T}} / \mathrm{FI}_{\mathrm{C}}$ for QB-ICA. Color version available online.

\section{Establishment of the Calibration Curve of FM-ICA and QB-ICA}

Under the optimal conditions, a standard curve for the FM/QB-ICA method was constructed by plotting the $\mathrm{B} / \mathrm{B}_{0}$ against the logarithm of AFM1 concentrations. Figure $5 \mathrm{~A}$ showed that FM-ICA exhibited a dynamic linear range $(50-600 \mathrm{pg} / \mathrm{mL})$ with a halfmaximal inhibitory concentration $\left(\mathbf{I C}_{50}\right)$ of $166 \mathrm{pg} / \mathrm{mL}$. Similarly, Figure 5B indicated that QB-ICA exhibited a dynamic linear range $(100-1,000 \mathrm{pg} / \mathrm{mL})$ with an $\mathrm{IC}_{50}$ of $349 \mathrm{pg} / \mathrm{mL}$. The 2 regression equations were $\mathrm{y}_{\mathrm{FM}}=$ $1.773-0.575 \operatorname{Ln}(\mathrm{x})\left(\mathrm{R}^{2}=0.9915\right)$ and $\mathrm{y}_{\mathrm{QB}}=2.394-$ $0.753 \operatorname{Ln}(\mathrm{x})\left(\mathrm{R}^{2}=0.9903\right)$. The LOD was calculated at 42.3 and $93.2 \mathrm{pg} / \mathrm{mL}$ following the method of Anfossi et al. (2013).

The LOD of FM-ICA was approximately 2.2 times lower than that of QB-ICA. The LOD of FM-ICA satisfied the European Union's AFM1 limited standards (50 $\mathrm{pg} / \mathrm{mL}$ ) in milk, whereas the LOD of QB-ICA satisfied the AFM1 limited standards of America and China $(500 \mathrm{pg} / \mathrm{mL})$.

\section{Concentration of FM and QB with the Same FI}

The optical intensity of probe is one of the most important parameters for sensitivity of ICA method. To evaluate the FI of the FM and the QB, a Hitachi F-4500 fluorescence spectrophotometer (Tokyo, Japan) was employed to detect the fluorescence intensities of water-soluble FM and $\mathrm{QB}$ with the maximum emission wavelength at 526 and $627 \mathrm{~nm}$, respectively. As shown in Figures 6, the FI of FM at concentration of 0.80 $\mathrm{nmol} / \mathrm{L}$ (dissolved in ultrapure water) was basically the same (about 5,000) as QB at $80.0 \mathrm{nmol} / \mathrm{L}$ (dissolved in ultrapure water). Hence, for same number of FM/ QB particles, the luminescence intensity of FM was presumably 100 times higher than that of QB. Thus, the fluorescence signal of the FM provides a promising 
A

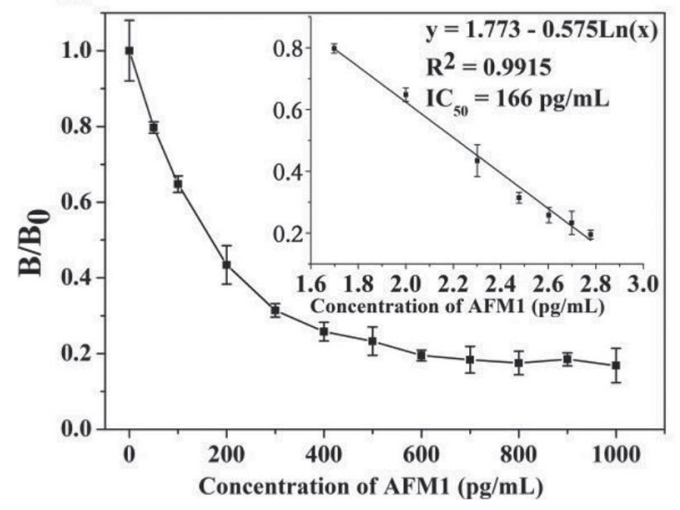

B

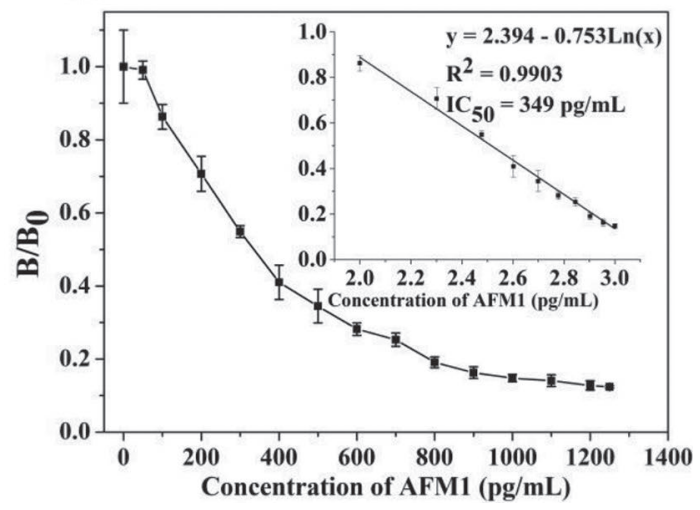

Figure 5. Results of quantitative detection of fluorescent microsphere immunochromatographic assay (FM-ICA) and quantum-dot submicrobead immunochromatographic assay (QB-ICA). A: Quantitative detection of aflatoxin $\mathrm{M}_{1}$ (AFM1) by FM-ICA. B: Quantitative detection of AFM1 by QB-ICA. Error bars indicate SD. B and $\mathrm{B}_{0}$ represent the fluorescent intensity of the T line/fluorescent intensity of the C line with and without AFM1 in the milk solutions, respectively. $\mathrm{IC}_{50}=$ half-maximal inhibitory concentration.

platform to improve the analytical sensitivity of FMICA.

\section{Accuracy and Precision of the 2 ICA Strips}

The FM-ICA recovery studies of the intraassays and interassays were conducted to evaluate the accuracy and precision of FM-ICA by analyzing 3 spiked milk samples with low, medium, and high AFM1 concentrations (Table 2). For FM-ICA, the average recoveries for the intraassays ranged from 82.04 to $121.02 \%$, with a CV ranging from 2.48 to $4.73 \%$. The average recoveries for the interassays ranged from 89.13 to $119.08 \%$, with a CV ranging from 4.33 to $6.16 \%$. In QB-ICA, the aver-

Table 1. Results of the checkerboard titration performed to optimize the parameters

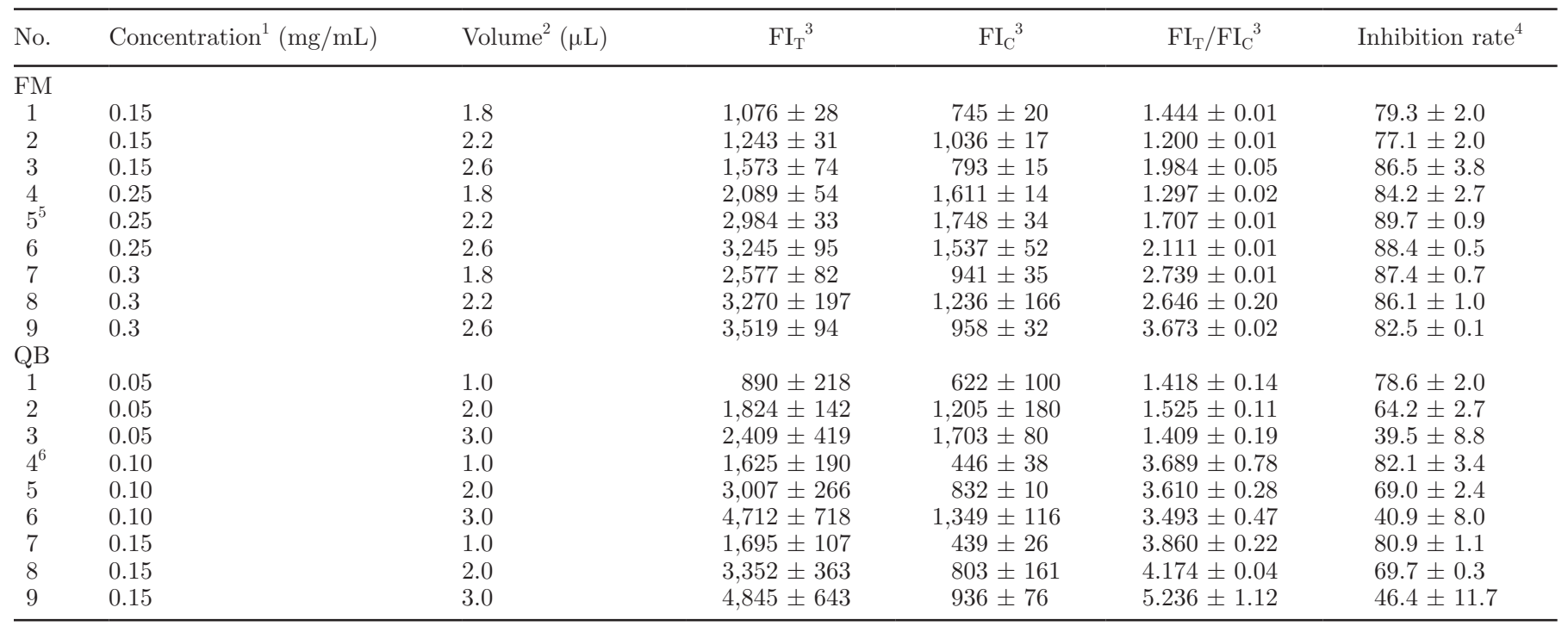

${ }^{1}$ Aflatoxin $\mathrm{M}_{1}$ (AFM1)-BSA concentration of fluorescent microsphere immunochromatographic assay (FM-ICA) or quantum-dot submicrobead immunochromatographic assay (QB-ICA).

${ }^{2}$ Probe volume of FM-monoclonal antibody $(\mathrm{mAb})$ probe or $\mathrm{QB}-\mathrm{mAb}$ probe.

${ }^{3} \mathrm{FI}_{\mathrm{T}}=$ fluorescent intensity of the $\mathrm{T}$ line; $\mathrm{FI}_{\mathrm{C}}=$ fluorescent intensity of the $\mathrm{C}$ line $( \pm \mathrm{SD})$.

${ }^{4}$ Inhibition rates $( \pm \mathrm{SD})$ were obtained from the 500 and $1,000 \mathrm{pg} / \mathrm{mL}$ concentrations of AFM1-spiked milk samples for FM-ICA and QB-ICA, respectively.

${ }^{5}$ Optimal parameters of FM-ICA.

${ }^{6}$ Optimal parameters of QB-ICA. 
Table 2. Accuracy and precision of the 2 immunochromatographic assay strips ${ }^{1}$

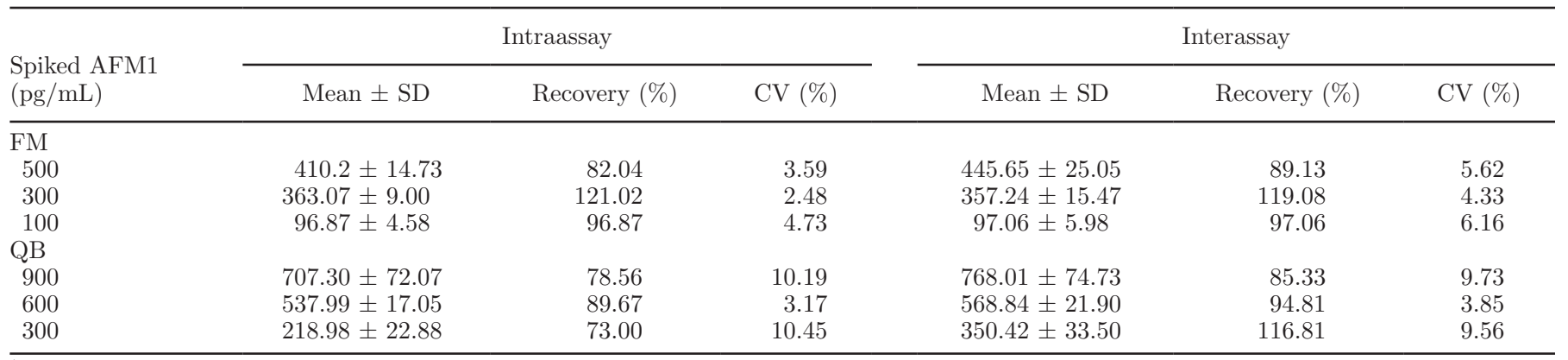

${ }^{1} \mathrm{AFM} 1=$ aflatoxin $\mathrm{M}_{1} ; \mathrm{FM}=$ fluorescent microsphere; $\mathrm{QB}=$ quantum-dot submicrobead.

age recoveries for the intraassay and interassay were 73.00 to $89.67 \%$ and 85.33 to $116.81 \%$, respectively, with corresponding CVs of 3.17 to $10.45 \%$ and 3.85 to $9.73 \%$.

\section{Reliability and Practicability of FM-ICA and QB-ICA}

To evaluate the reliability and practicability of FMICA and QB-ICA, the 2 methods were compared with LC-MS/MS. Negative milk samples spiked with AFM1 at 50,250 , and $500 \mathrm{pg} / \mathrm{mL}$ were tested using FM-ICA and LC-MS/MS. Negative milk samples spiked with AFM1 at 200, 500, and $800 \mathrm{pg} / \mathrm{mL}$ were tested using QB-ICA and LC-MS/MS.

As shown in Table 3, the linear correlation equation between FM-ICA and LC-MS/MS was $\mathrm{y}=1.08 \mathrm{x}-$ 1.43 with a reliable correlation coefficient $\left(\mathrm{R}^{2}=0.994\right)$, and the linear correlation equation between QB-ICA and LC-MS/MS was $\mathrm{y}=0.89 \mathrm{x}+35.52$ with a reliable correlation coefficient $\left(\mathrm{R}^{2}=0.967\right)$.

\section{Specificity of FM-ICA and QB-ICA}

To evaluate their specificity, FM-ICA and QB-ICA were tested with AFM1 and 4 aflatoxins, namely, DON,
AFB1, OTA, and ZEN at concentrations of $10 \mathrm{ng} / \mathrm{mL}$. As shown in Figure 7 , the $\mathrm{FI}_{\mathrm{T}} / \mathrm{FI}_{\mathrm{C}}$ of DON, OTA, and ZEN were as high as those of the negative samples. This result indicated that FM-ICA and QB-ICA lack immune responses to DON, OTA, and ZEN. However, the $\mathrm{FI}_{\mathrm{T}} / \mathrm{FI}_{\mathrm{C}}$ of AFB1 was 0 because AFM1 is a major monohydroxylated derivative of AFB1. Thus, FM-ICA and QB-ICA can specifically detect AFM1.

\section{Validation of the Methods for Practical Use}

The 12 raw milk samples were detected using FMICA, QB-ICA, and LC-MS/MS. The result was presented in Table 4. The concentration of AFM1 in 12 milk samples was lower than the LOD of FM-ICA and QB-ICA. The detection results of the FM-ICA and QBICA were in good agreement with that of the LC-MS/ MS method.

\section{CONCLUSIONS}

Systematic and comprehensive comparison between novel FM-ICA and QB-ICA in quantitative detection of AFM1 in milk was studied for the first time. The advantages of FM-ICA include lower LOD of $42.3 \mathrm{pg} / \mathrm{mL}$,

Table 3. Correlation between results from (x-axis) liquid chromatography-tandem mass spectrometry (LC-MS/MS) and quantum-dot submicrobead immunochromatographic assay (QB-ICA)/fluorescent microsphere immunochromatographic assay (FM-ICA; y-axis) analyses of aflatoxin $\mathrm{M}_{1}$ (AFM1) in spiked milk samples

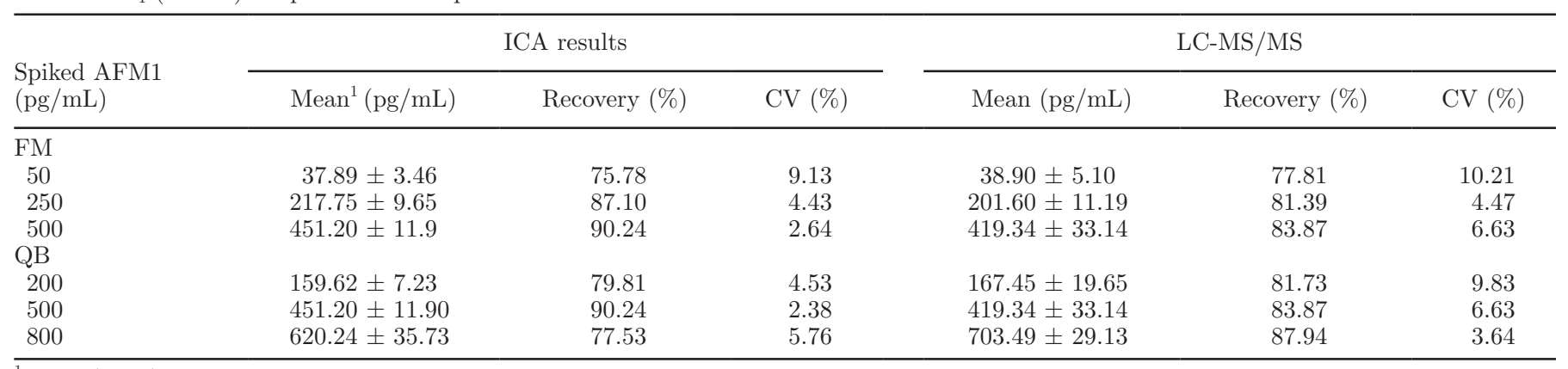

${ }^{1}$ Mean $( \pm \mathrm{SD})$ value of 3 replicates at each spiked concentration. 


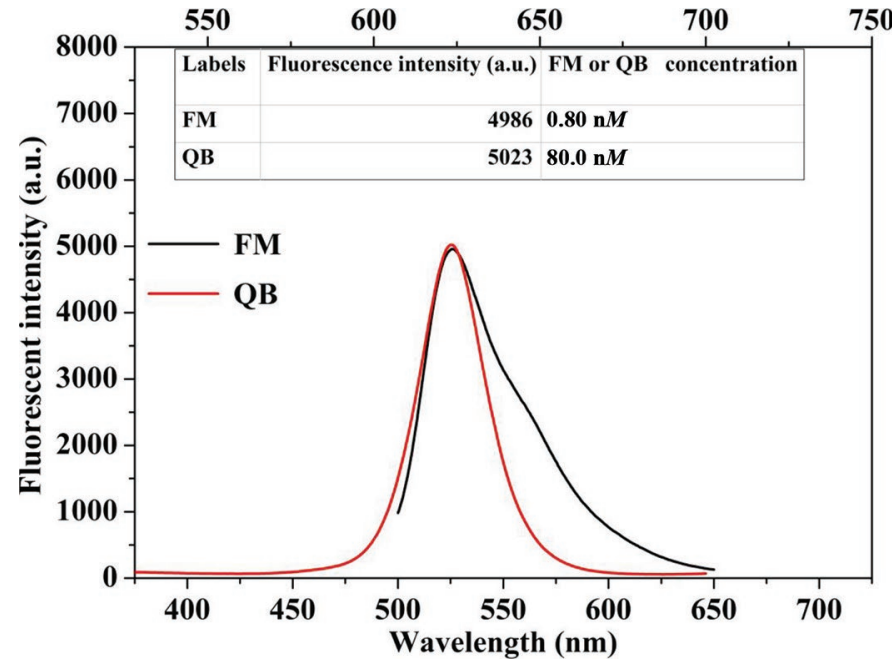

Figure 6. Photoluminescence spectra of fluorescent microsphere $(\mathrm{FM} ; 0.80 \mathrm{nmol} / \mathrm{L}$, dissolved in ultrapure water) and quantum-dot submicrobead (QB; $80.0 \mathrm{nmol} / \mathrm{L}$, dissolved in ultrapure water). a.u. = arbitrary units. Color version available online.

better accuracy and precision, and better reliability and practicability. The advantages of QB-ICA include short detection time and low mAb consumption. This study serves as a reference for selecting appropriate fluorescent labels for the ICA of AFM1 in milk.

\section{ACKNOWLEDGMENTS}

This work was financially supported by the Jiangxi Education Bureau Technology Project (KJLD13009, China) and the Jiangxi Technological Program (20141BBF60042, China).

Table 4. Detection results of raw milk samples ${ }^{1}$

\begin{tabular}{lccc}
\hline & \multicolumn{3}{c}{ Detection results $(\mathrm{pg} / \mathrm{mL})$} \\
\cline { 2 - 4 } Sample & FM-ICA & QB-ICA & LC-MS/MS \\
\hline 1 & ND & ND & ND \\
2 & ND & ND & ND \\
3 & ND & ND & ND \\
4 & ND & ND & 5.12 \\
5 & ND & ND & 3.04 \\
6 & ND & ND & ND \\
7 & ND & ND & ND \\
8 & ND & ND & ND \\
9 & ND & ND & ND \\
10 & ND & ND & 0.86 \\
11 & ND & ND & 1.16 \\
12 & ND & ND & ND \\
\hline
\end{tabular}

${ }^{1} \mathrm{FM}-\mathrm{ICA}=$ fluorescent microsphere immunochromatographic assay; QB-ICA = quantum-dot submicrobead immunochromatographic assay; LC-MS/MS = liquid chromatography-tandem mass spectrometry; $\mathrm{ND}=$ not detected.

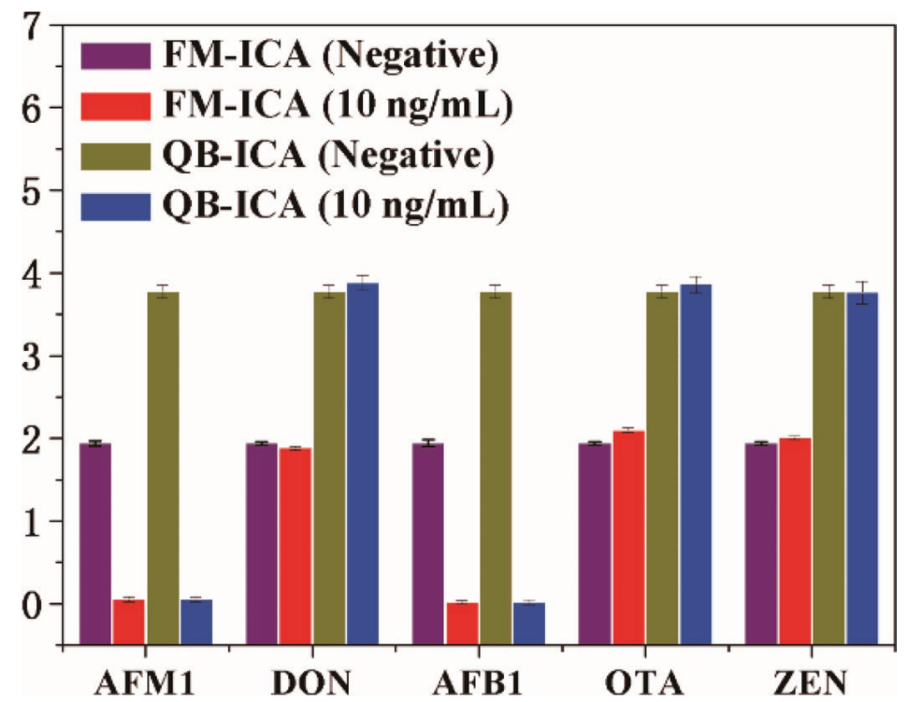

Figure 7. Specificity experiment of fluorescent microsphere immunochromatographic assay (FM-ICA) and quantum-dot submicrobead immunochromatographic assay (QB-ICA) for $10 \mathrm{ng} / \mathrm{mL}$ of aflatoxin $\mathrm{M}_{1}$ (AFM1), deoxynivalenol (DON), aflatoxin $\mathrm{B}_{1}$ (AFB1), ochratoxin A (OTA), and zearalenone (ZEN). Error bars indicate SD. Color version available online.

\section{REFERENCES}

Anfossi, L., C. Baggiani, C. Giovannoli, F. Biagioli, G. D'Arco, and G. Giraudi. 2013. Optimization of a lateral flow immunoassay for the ultrasensitive detection of aflatoxin M 1 in milk. Anal. Chim. Acta 772:75-80.

Busman, M., J. R. Bobell, and C. M. Maragos. 2015. Determination of the aflatoxin M 1 (AFM 1) from milk by direct analysis in real time-mass spectrometry (DART-MS). Food Contr. 47:592-598.

Chen, R., H. Li, H. Zhang, S. Zhang, W. Shi, J. Shen, and Z. Wang. 2013a. Development of a lateral flow fluorescent microsphere immunoassay for the determination of sulfamethazine in milk. Anal. Bioanal. Chem. 405:6783-6789.

Chen, Y., Y. Xin, H. Yang, L. Zhang, Y. Zhang, X. Xia, Y. Tong, and W. Wang. 2013b. Immobilization and stabilization of cholesterol oxidase on modified sepharose particles. Int. J. Biol. Macromol. 56:6-13.

Du, B., X. Su, K. Yang, L. Pan, Q. Liu, L. Gong, P. Wang, J. Yang, and Y. He. 2016. Antibody-free colorimetric detection of total aflatoxins in rice based on a simple two-step chromogenic reaction. Anal. Chem. 88:3775-3780.

Duan, H., X. Chen, W. Xu, J. Fu, Y. Xiong, and A. Wang. 2015. Quantum-DoT submicrobead-based immunochromatographic assay for quantitative and sensitive detection of zearalenone. Talanta 132:126-131.

Ensafi, A. A., N. Kazemifard, and B. Rezaei. 2016. A simple and sensitive fluorimetric aptasensor for the ultrasensitive detection of arsenic (III) based on cysteamine stabilized $\mathrm{CdTe} / \mathrm{ZnS}$ quantum dots aggregation. Biosens. Bioelectron. 77:499-504.

GB 5413.37. 2010. Determination of aflatoxin M1 in milk and milk products. National Food Safety Standard, China.

Guo, X., F. Wen, N. Zheng, Q. Luo, H. Wang, H. Wang, S. Li, and J. Wang. 2014. Development of an ultrasensitive aptasensor for the detection of aflatoxin B 1. Biosens. Bioelectron. 56:340-344.

Hu, J., Z.-L. Zhang, C.-Y. Wen, M. Tang, L.-L. Wu, C. Liu, L. Zhu, and D.-W. Pang. 2016. Sensitive and quantitative detection of Creaction protein based on immunofluorescent nanospheres coupled with lateral flow test strip. Anal. Chem. 88:6577-6584. 
Huang, S., D. Hu, Y. Wang, F. Zhu, R. Jiang, and G. Ouyang. 2015. Automated hollow-fiber liquid-phase microextraction coupled with liquid chromatography/tandem mass spectrometry for the analysis of aflatoxin M 1 in milk. J. Chromatogr. A 1416:137-140.

Huang, X., Z. P. Aguilar, H. Li, W. Lai, H. Wei, H. Xu, and Y. Xiong. 2013. Fluorescent Ru (phen) 32+-doped silica nanoparticles-based ICTS sensor for quantitative detection of enrofloxacin residues in chicken meat. Anal. Chem. 85:5120-5128.

Li, X., W. Li, Q. Yang, X. Gong, W. Guo, C. Dong, J. Liu, L. Xuan, and J. Chang. 2014. Rapid and quantitative detection of prostate specific antigen with a quantum dot nanobeads-based immunochromatography test strip. ACS Appl. Mater. Interfaces 6:64066414.

Liu, D., Y. Huang, M. Chen, S. Wang, K. Liu, and W. Lai. 2015. Rapid detection method for aflatoxin B 1 in soybean sauce based on fluorescent microspheres probe. Food Contr. 50:659-662.

Lu, S., G. Li, Z. Lv, N. Qiu, W. Kong, P. Gong, G. Chen, L. Xia, X. Guo, and J. You. 2016. Facile and ultrasensitive fluorescence sensor platform for tumor invasive biomaker $\beta$-glucuronidase detection and inhibitor evaluation with carbon quantum dots based on inner-filter effect. Biosens. Bioelectron. 85:358-362.

Nankoberanyi, S., G. W. Mbogo, N. P. LeClair, M. D. Conrad, P. Tumwebaze, S. Tukwasibwe, M. R. Kamya, J. Tappero, S. L. Nsobya, and P. J. Rosenthal. 2014. Validation of the ligase detection reaction fluorescent microsphere assay for the detection of Plasmodium falciparum resistance mediating polymorphisms in Uganda. Malar. J. 13:95.

Ossa, D. E. H., D. A. Hincapié, and G. A. Peñuela. 2015. Determination of aflatoxin M 1 in ice cream samples using immunoaffinity columns and ultra-high performance liquid chromatography coupled to tandem mass spectrometry. Food Contr. 56:34-40.

Ren, M., H. Xu, X. Huang, M. Kuang, Y. Xiong, H. Xu, Y. Xu, H. Chen, and A. Wang. 2014. Immunochromatographic assay for ultrasensitive detection of aflatoxin B1 in maize by highly luminescent quantum dot beads. ACS Appl. Mater. Interfaces 6:1421514222 .

Shan, S., W. Lai, Y. Xiong, H. Wei, and H. Xu. 2015. Novel strategies to enhance lateral flow immunoassay sensitivity for detecting foodborne pathogens. J. Agric. Food Chem. 63:745-753.

Shen, J., Y. Zhou, F. Fu, H. Xu, J. Lv, Y. Xiong, and A. Wang. 2015. Immunochromatographic assay for quantitative and sensitive detection of hepatitis B virus surface antigen using highly luminescent quantum dot-beads. Talanta 142:145-149.
Taherimaslak, Z., M. Amoli-Diva, M. Allahyary, and K. Pourghazi. 2014. Magnetically assisted solid phase extraction using $\mathrm{Fe}_{3} \mathrm{O}_{4}$ nanoparticles combined with enhanced spectrofluorimetric detection for aflatoxin M1 determination in milk samples. Anal. Chim. Acta 842:63-69.

Vdovenko, M. M., C.-C. Lu, F.-Y. Yu, and I. Y. Sakharov. 2014. Development of ultrasensitive direct chemiluminescent enzyme immunoassay for determination of aflatoxin M1 in milk. Food Chem. 158:310-314.

Wang, H., H. Wang, S. Chen, E. E. Dzakah, K. Kang, J. Wang, and J. Wang. 2015. Development of a fluorescent immnunochromatographic assay for the procalcitonin detection of clinical patients in China. Clin. Chim. Acta 444:37-42.

Wang, Z., H. Li, C. Li, Q. Yu, J. Shen, and S. De Saeger. 2014. Development and application of a quantitative fluorescence-based immunochromatographic assay for fumonisin B1 in maize. J. Agric. Food Chem. 62:6294-6298.

Xie, Q.-Y., Y.-H. Wu, Q.-R. Xiong, H.-Y. Xu, Y.-H. Xiong, K. Liu, Y. Jin, and W.-H. Lai. 2014. Advantages of fluorescent microspheres compared with colloidal gold as a label in immunochromatographic lateral flow assays. Biosens. Bioelectron. 54:262-265.

Yang, Y.. C. Li, W. Wang, T. Dong, Y. Xiong, J. Shen, and W. Lai. 2015. A fluorescence immunochromatographic assay for rapid and sensitive detection of human prealbumin in serum. Anal. Methods $7: 8683-8688$

Zhang, G., M. Chen, D. Liu, Y. Xiong, R. Feng, P. Zhong, and W. Lai. 2016. Quantitative detection of $\beta$ 2-adrenergic agonists using fluorescence quenching by immunochromatographic assay. Anal. Methods 8:627-631.

Zhang, X., C. Wu, K. Wen, H. Jiang, J. Shen, S. Zhang, and Z. Wang. 2015. Comparison of fluorescent microspheres and colloidal gold as labels in lateral flow immunochromatographic assays for the detection of T-2 toxin. Molecules 21:E27.

Zhang, Z., Y. Li, P. Li, Q. Zhang, W. Zhang, X. Hu, and X. Ding. 2014. Monoclonal antibody-quantum dots CdTe conjugate-based fluoroimmunoassay for the determination of aflatoxin B 1 in peanuts. Food Chem. 146:314-319.

Zhou, J., K. Zhu, F. Xu, W. Wang, H. Jiang, Z. Wang, and S. Ding. 2014. Development of a microsphere-based fluorescence immunochromatographic assay for monitoring lincomycin in milk, honey, beef, and swine urine. J. Agric. Food Chem. 62:12061-12066. 